Supporting Information:

\title{
Black Phosphorus Nanosheet/Melamine Cyanurate Assemblies as Functional Active Layers for Artificial Synapse Memristors
}

\author{
Yu-Ling Hsieh ${ }^{\dagger}$ and Ching-Yuan $\mathrm{Su}^{\dagger \# \% *}$ \\ † Dep. of Mechanical Engineering, National Central University, Tao-Yuan 32001, Taiwan \\ \# Graduate Institute of Energy Engineering, National Central University, Tao-Yuan 32001, \\ Taiwan \\ ${ }^{\%}$ Graduate Institute of Material Science and Engineering, National Central University, Tao- \\ Yuan 32001, Taiwan
}

To whom correspondence should be addressed: (C. Y. Su) cysu@ncu.edu.tw

KEYWORDS: Black Phosphorus (BP), liquid-phase exfoliation (LPE), 2D materials, supramolecular assembly, resistive switching, memristor, selector 


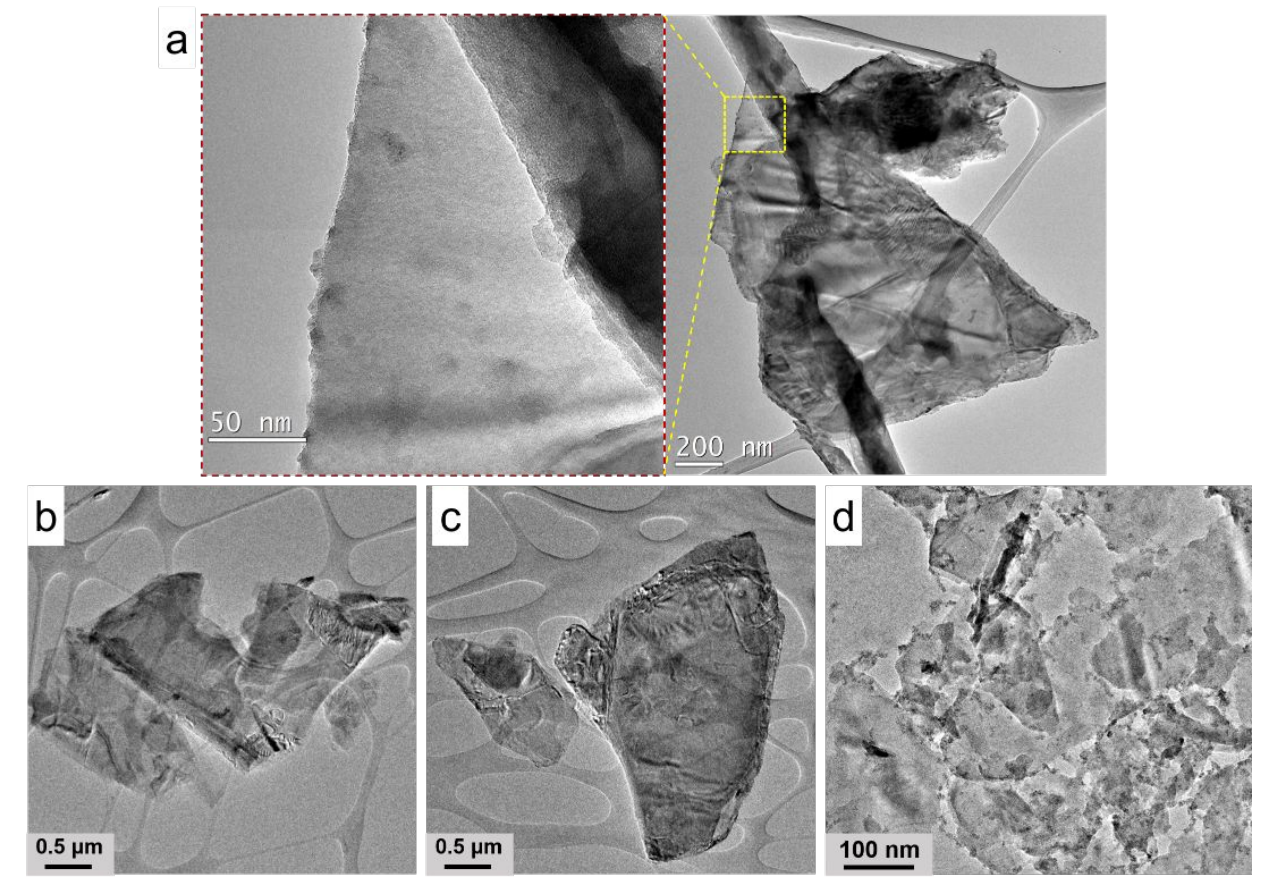

Figure S1. TEM images of as-exfoliated BP sheets with different magnification: (a) The enlarged TEM image at the area of thin BP sheet. (b)-(d) The separated BP sheets with wide-view observation.

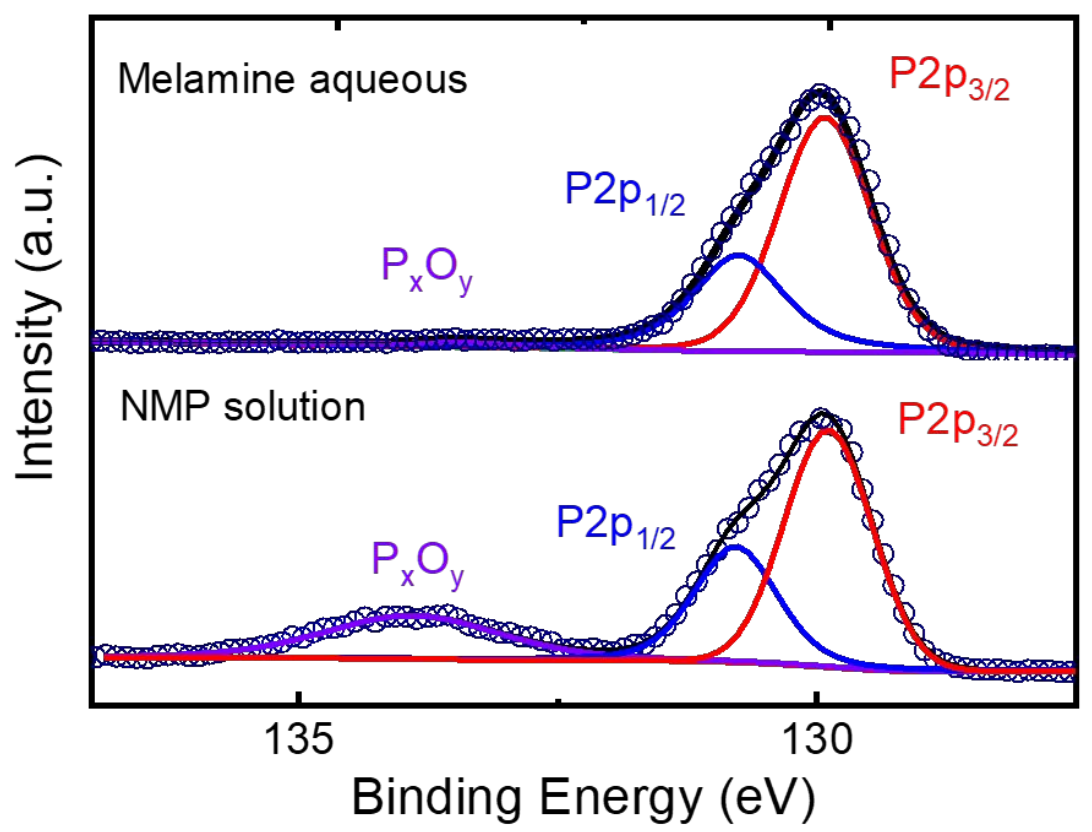

Figure S2. XPS spectra of liquid-phase exfoliated BP sheets by using melamine aqueous and NMP solutions. 

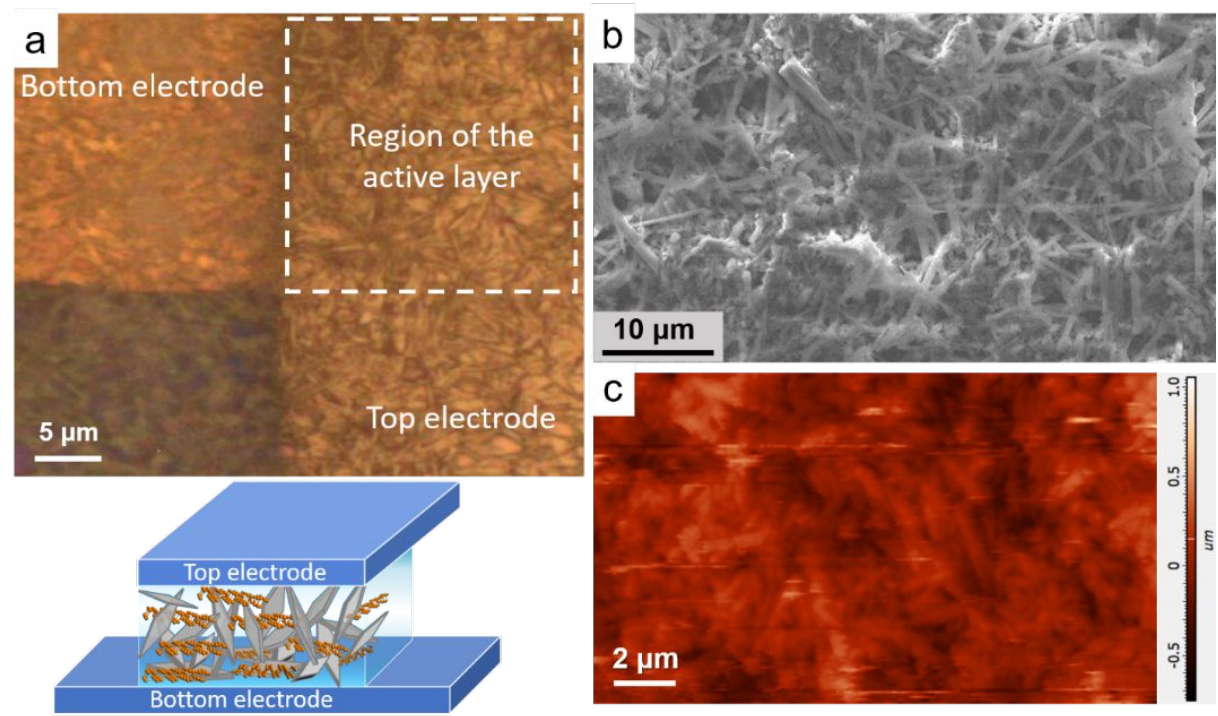

Figure S3. (a) Optical microscope image of the memristor, where the region of the active layer is marked by dashed square. (bottom: Schematic illustration of the two-terminal structure of the memristor. (b) SEM and (c) AFM images of the active layer of MCA supramolecules.

Table S1. Comparison of 2D material-based memristor devices.

\begin{tabular}{|c|c|c|c|c|c|c|c|c|}
\hline $\begin{array}{l}\text { Material of } \\
\text { active layer }\end{array}$ & $\begin{array}{l}\text { Electrodes/ } \\
\text { Substrate }\end{array}$ & $\begin{array}{l}\text { Stimulus } \\
\text { signal }\end{array}$ & $\begin{array}{l}\text { Spike } \\
\text { width }\end{array}$ & Working Principle & $\begin{array}{l}\text { Synaptic/ } \\
\text { neuronal } \\
\text { functions }\end{array}$ & Device size & Scalability & Reference \\
\hline $\begin{array}{l}\text { BP:MCA } \\
\text { supramolecules }\end{array}$ & $\begin{array}{l}\text { Top: } \mathrm{Au} \\
\text { Bottom: } \mathrm{Au} \\
{\mathrm{On} \mathrm{SiO}_{2} / \mathrm{Si}}\end{array}$ & $0.5 \mathrm{~V}$ & $5 \mathrm{~ms}$ & Proton transfer & LTP/ LTD & $\begin{array}{l}50 \mu \mathrm{m} \times 50 \\
\mu \mathrm{m}\end{array}$ & Yes & This work \\
\hline $\begin{array}{l}\text { BP } \\
\text { nanosheets@PS }\end{array}$ & $\begin{array}{l}\text { Top: ITO } \\
\text { Bottom: ITO } \\
\text { On Glass }\end{array}$ & $\begin{array}{l}-0.5 \mathrm{~V} \text { with } \\
\text { light } \\
\text { illumination }\end{array}$ & NA & $\begin{array}{l}\text { Charge trapping/ } \\
\text { detrapping }\end{array}$ & $\begin{array}{l}\text { Enhanced } \\
\text { resistive } \\
\text { switching }\end{array}$ & $\begin{array}{l}\text { Diameter of } \\
200 \mu \mathrm{m}\end{array}$ & Yes & 1 \\
\hline $\mathrm{P}_{\mathrm{x}} \mathrm{O}_{\mathrm{y}} / \mathrm{BP}$ flake & $\begin{array}{l}\text { (Parallel) } \\
\mathrm{Au} / \mathrm{Cr} \\
\mathrm{On} \mathrm{SiO}_{2} / \mathrm{Si}\end{array}$ & $\begin{array}{l}\text { Optical pulses } \\
280 \mathrm{~nm}, 365 \\
\mathrm{~nm}\end{array}$ & $\begin{array}{l}1,20,50 \\
100, \text { and } \\
300 \mathrm{~ms}\end{array}$ & $\begin{array}{l}\text { Charge trapping/ } \\
\text { detrapping }\end{array}$ & LTP/ LTD & $\begin{array}{l}\sim 3 \mu \mathrm{m} \times 2 \\
\mu \mathrm{m}\end{array}$ & No & 2 \\
\hline $\begin{array}{l}\text { SnSe/BP } \\
\text { heterojunction }\end{array}$ & $\begin{array}{l}\text { Top: } \mathrm{Au} \\
\text { Bottom: } \mathrm{Au} \\
{\mathrm{On} \mathrm{SiO}_{2} / \mathrm{Si}}\end{array}$ & $20 \mathrm{~V}$ & $10 \mathrm{~s}$ & $\begin{array}{l}\mathrm{n} / \mathrm{p} \text { junction tunning; } \\
\text { Charge trapping/ } \\
\text { detrapping }\end{array}$ & $\begin{array}{l}\text { STDP } \\
\text { LTP/ LTD }\end{array}$ & $\begin{array}{l}\sim 10 \mu \mathrm{m} \times 7 \\
\mu \mathrm{m}\end{array}$ & No & 3 \\
\hline hBN flake & 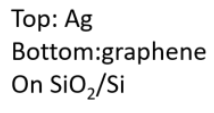 & $1.2 \mathrm{~V}$ & $75 \mathrm{~ms}$ & Ag ions diffusion & LTP/ LTD & $\begin{array}{l}\text { Few } \\
\text { micrometers }\end{array}$ & No & 4 \\
\hline $\mathrm{MoS}_{2}$ ink & $\begin{array}{l}\text { Top: Ag } \\
\text { Bottom: Ag } \\
\text { On Polymide }\end{array}$ & $1.2 \mathrm{~V} /-0.1 \mathrm{~V}$ & $1 \mathrm{~ms}$ & Ag ions diffusion & $\begin{array}{l}\text { STDP } \\
\text { LTP/ LTD }\end{array}$ & $\begin{array}{l}100 \mu \mathrm{m} x \\
100 \mu \mathrm{m}\end{array}$ & Yes & 5 \\
\hline
\end{tabular}




\section{References:}

(1) Zhou, Y.; Liu, D.; Wang, J.; Cheng, Z.; Liu, L.; Yang, N.; Liu, Y.; Xia, T.; Liu, X.; Zhang, X.; Ye, C.; Xu, Z.; Xiong, W.; Chu, P. K.; Yu, X.-F., Black Phosphorus Based Multicolor Light-Modulated Transparent Memristor with Enhanced Resistive Switching Performance. ACS Appl. Mater. Interfaces 2020, 12 (22), 25108-25114. DOI: 10.1021/acsami.0c04493

(2) Ahmed, T.; Tahir, M.; Low, M. X.; Ren, Y.; Tawfik, S. A.; Mayes, E. L. H.; Kuriakose, S.; Nawaz, S.; Spencer, M. J. S.; Chen, H.; Bhaskaran, M.; Sriram, S.; Walia, S. Fully Light-Controlled Memory and Neuromorphic Computation in Layered Black Phosphorus. Adv. Mater. 2020, 2004207. DOI: 10.1002/adma.202004207

(3) Tian, H.; Cao, X.; Xie, Y.; Yan, X.; Kostelec, A.; DiMarzio, D.; Chang, C.; Zhao, L.-D.; Wu, W.; Tice, J.; Cha, J. J.; Guo, J.; Wang, H. Emulating Bilingual Synaptic Response Using a Junction-Based Artificial Synaptic Device. ACS Nano 2017, 11 (7), 7156-7163. DOI: $10.1021 /$ acsnano.7b03033

(4) Sun, L.; Hwang, G.; Choi, W.; Han, G.; Zhang, Y.; Jiang, J.; Zheng, S.; Watanabe, K.; Taniguchi, T.; Zhao, M.; Zhao, R.; Kim, Y.-M.; Yang, H. Ultralow Switching Voltage Slope Based on Two-Dimensional Materials for Integrated Memory and Neuromorphic Applications. Nano Energy 2020, 69, 104472. DOI: https://doi.org/10.1016/j.nanoen.2020.104472

(5) Feng, X.; Li, Y.; Wang, L.; Chen, S.; Yu, Z. G.; Tan, W. C.; Macadam, N.; Hu, G.; Huang, L.; Chen, L.; Gong, X.; Chi, D.; Hasan, T.; Thean, A. V.-Y.; Zhang, Y.-W.; Ang, K.-W. A Fully Printed Flexible MoS2 Memristive Artificial Synapse with Femtojoule Switching Energy. Adv. Electron. Mater. 2019, 5 (12), 1900740. DOI:

10.1002/aelm.201900740 